\title{
Communication and Ubiquitous Computing
}

\author{
Sun-Moon Jo' $\mathrm{Ju}^{1}$ Yu-Keum Jeong ${ }^{2} \cdot \mathrm{Jungsoo} \mathrm{Han}^{3}$
}

Published online: 14 November 2015

(C) Springer Science+Business Media New York 2015

Welcome to this special issue of Wireless Personal Communications. This issue contains a collection of the best papers out of various authors who have been submitted to this issue. The main goal for this issue is to be a timely vehicle for publishing selected research papers from practitioners and academia in convergence industries on this emerging topic. This issue covers some of the hottest topics in Communication and Ubiquitous Computing, including: applications for interaction processing; networking and architectures; information indexing, searching, and visualization; intelligent information processing algorithms; sensors, wireless technology and embedded systems; converged and interactive applications and frameworks; future communication systems; hybrid networking system; multicasting and multimedia delivery; architectures and protocols; data and index structures; semantic routing and search.

The paper by Jeong and Shin [1] proposes an efficient authentication scheme to protect user privacy in seamless big data services. The proposed method is a security management scheme that allows users to easily access big data from different network environments by assigning a key shared among users and servers, linking big data and users' attribute information. The paper by Lee et al. [2] presents a systematic classification management scheme to

Jungsoo Han

jungsoo.han.k@gmail.com

Sun-Moon Jo

sunmoon@pcu.ac.kr

Yu-Keum Jeong

digital@policy.or.kr

1 Department of Computer Information Technology Education, Paichai University, Doma 2-dong, Seo-gu, Daejeon, Republic of Korea

2 Strategy Department, Society of Digital Policy and Management, Hicetown PRIME 306, Seongjeongdujeong-ro 71, Cheonan-si, Chungcheongnam-do, Republic of Korea

3 Division of Information and Communication, Baekseok University, 76, Munam-ro, Anseo-dong, Dongnam-gu, Cheonan-si, Chungcheongnam-do, Republic of Korea 
provide an efficient peer-to-peer national research and development service. They suggest aspects of a systematic subject classification scheme for national R\&D information, to more systematically manage national R\&D information, to further analyze it, and provide it more efficiently. This system-based management will contribute to easy management and systematic provision of explosively increased information in various fields.

The paper by Lee and Lee [3] proposes alpha-cut filter-based proxy caching (AFPC) control for wireless coverage streaming services. The proposed method is a novel mechanism for wireless coverage streaming services. AFPC consists of a burst-referenced caching strategy (BRCS) and a capacity-referenced caching strategy (CRCS) to acquire the optimal streaming quality for continuous objects and non-continuous objects. BRCS controls streaming congestion by considering the encoding rate and channel bandwidth; the CRCS scheme reduces caching congestion by scheduling different types of stream packets based on the restricted caching resource and media characteristics. The paper by Lee and Seo [4] introduces a hybrid multi-criteria decision-making model for a cloud service selection problem using balanced scorecard (BSC), a fuzzy Delphi method, and a fuzzy analytical hierarchy process (AHP). They are applied to define the major hierarchy and to derive decision-making criteria and decision-making factors selected for each BSC perspective. It is used to select the best cloud service from among cloud service alternatives based on predetermined decision-making criteria and factors.

The paper by Lee et al. [5] proposes a study on the improved normalized cut algorithm using a bilateral filter for efficient object extraction from an image. The proposed method is a normalized cut algorithm using a bilateral filter for fast transmission in an intelligent traffic control system. They modified a standard normalized cut algorithm to improve object extraction from noisy or complex images by applying a bilateral filter to binarize them, extracting outlines using the canny edge method and applying a dilation operation. This algorithm reduced the processing time for extracting objects, allowing much faster data transmission. The paper by Kim et al. [6] proposes a mobile-based denial-of-service (DoS) attack security agent in sensor networking. The proposed mobile-based security agent provides security mechanisms to security agents as well as hosts. This type of security agent is significant in providing a security mechanism against security risks in cloud computing due to its ability to migrate from one host to another, where it can resume its processing and service provisioning. Fault tolerance at a high level was enabled for mobile-based distributed transaction processing in the cloud.

The paper by Jang and Jung [7] proposes detection of harmful content using multilevel verification in visual sensor data. They show an algorithm for extracting human nipple regions, representing the harmfulness of the images, using a multilevel verification technique in visual sensor-based image data. The proposed algorithm detects human face regions, including eyes and lips, from input images. They select real nipple areas after eliminating non-nipple regions at multiple levels by applying geometric information and an average color filter to the detected candidate nipple regions. The paper Lee et al. [8] proposes an adaptive asymmetric transmission scheme for MPEG4/SVC signals in MIMOOFDM systems. The proposed system has to preferentially protect the base layer to compose the SVC signal, and the remaining enhanced layers are protected in order to meet video quality requirements. It is possible to lessen the effects of the channel environment, thereby ensuring video quality. Each layer has a different data size, which makes it possible to implement differential channel coding.

The paper by Choi and Sim [9] proposes a study on the efficiency of markup language using DOM tree. The proposed method uses DOM tree extension with the advantage of fast accessibility for XML documents. Simple and complex DOM tree uses an XML schema of 
the DOM base and offers variety in creating a markup language. The markup language generation of MetaData that use the DOM form improves the reusability ratio of the markup language, because it is possible to provide detailed data type expression and classified unit elements. The paper by Lee et al. [10] shows the development of a lighting control system based on context-awareness for the improvement of learning efficiency in the classroom. The proposed method is a standard index for lighting illumination that is effective for concentration, depending on the subjects. LED lighting is produced where color temperature, intensity of illumination, and the on/off switch are adjustable, in addition to providing hybrid sensors for context awareness. Based on the learning schedule defined in advance and data collected through sensors, a context-awareness process is created to grasp the current class context.

The paper by Kim et al. [11] proposes slope-based intelligent 3D disaster simulation using a physics engine. The developed method infers a variety of disaster situations that may occur in reality through ontology and applies it to 3D simulation. Disaster situation ontology constitutes context information about outside and inside disasters, etc., and generates a rule base using a Jena 2.0 inference engine. Inference results are derived in the form of XML and are applicable to a variety of devices. The simulation operates object collision, movement, and surroundings, etc., in real time by using a physics engine, and represents a disaster situation in 3D virtual space by using a particle function. Environment context information on slopes, including landslides, snow, rain, fog, fire, explosion, and smoke, is developed in the slope-based intelligent 3D disaster simulation. The paper by Lee and Lee [12] proposes a dynamic bio-sensing process design in a mobile wellness information system for smart healthcare. The proposed method is a customized healthcare service by means of wellness clothing that includes digital yarns and bio-sensors. Wellness clothing is utilized to acquire, analyze, and present bio-engineering data, including ECG, respiration, acceleration, and body temperature, as part of the wellness information system framework. The conventional process configuration of a biometric information system performs the fixed process without changing statically and consistently after the system starts.

The paper by Park et al. [13] proposes a study on factors affecting the adoption of LTE mobile communications services. They find the factors affecting the intention to adopt LTE services through a survey conducted in South Korea. This study focused on the functional dimension, including cost, speed, and security, the social dimension, including social influence, and the personal dimension, including personal innovativeness and self-efficacy to find out their effects on intention to accept LTE services. People tended to use LTE because it is more effective for interacting with one another (social influence and speed appeared to be significant in the model). The improvement in convenience seems to be useful in increasing the rate of LTE adoption. The paper by Park et al. [14] proposes a case study for an effective technique of distributed data storage for big data processing in the wireless Internet environment. The proposed method presents the establishment of a cloud storage-based file system that can provide services to meet the needs of users in the cloud computing environment via wireless Internet, plus examples of the establishment of such a system. They analyzed the performance of local and overseas SAN systems. Cost effectiveness was employed as the performance measure for analysis.

The paper by Kim et al. [15] proposes continuous gesture recognition using higher order local auto correlation (HLAC) and low-dimensional space. The proposed method presents a separate two-dimensional silhouette gesture region from a continuous input image that includes a human body image. Information about 35 features are extracted using the HLAC coefficient in the divided image, and a low-dimensional gesture space is composed using principal component analysis. The model feature value reflected in the gesture space is 
composed of symbols of certain conditions through a clustering algorithm, so as to be used as the input symbol of a hidden marker model. Random input motion is recognized as the relevant gesture model with the highest probability value. The paper by Yang et al. [16] predicts a second-language proficiency level using a linguistic cognitive task and machine learning techniques. The proposed method uses machine learning with a linguistic cognitive ability measured through cognitive tasks, rather than using the indices from conventional language proficiency test scores and learning systems. They predict language proficiency by looking into the cognitive processes in the human brain. The biggest advantage over conventional language proficiency tests is that it does not require as much time, cost, or a large amount of data collected from the learner.

The paper by Kim and $\mathrm{Na}$ [17] introduces a safe data transmission architecture based in the cloud for the Internet of Things (IoT). The proposed safe data transmission architecture (S-DTA) builds a safe network via synchronization-based software-defined network technology for an efficient cloud-based IoT ecosystem. It is designed to identify the status of the various data to be transmitted based on M2M/IoT technology with QoS/QoE, and it is possible to ensure transmission of more transparent and secure data. The paper by Jeong and Cho [18] shows a digitalized recomposition technique based on photo quality-evaluation criteria. The proposed method uses rule-of-thirds as an optimization problem of the feature vector in an image. Simplicity factors can be formulated as a calculation problem for the size of the region of interest (ROI) segments. The size of the ROI and the moving direction of the foreground object are used to formulate the rule of space. The presented algorithm can be applied in the near future, because many related state-of-the-art technologies are embedded in commercial cameras.

The paper by Jung and Chung [19] proposes a PHR-based life health index mobile service using a decision support model. They present multivariate analysis for health index-related factors based on data of the national health and nutrition survey and decision support modeling. In order to serve the user's health index according to the decision support model, a mobile application was developed and implemented for easy management and services in mobile applications by using PHR information to manage user information easily. The developing health service using the decision support model is expected to contribute to health promotion by providing effective services to users in U-health environments. The paper by Yang and Sun [20] discusses a study on stable data transmission using a hierarchical share group in a mobile ad hoc network. The proposed method is a hierarchical sharing group configuration technique to provide a stable connection between the mobile nodes and to reduce the load of network traffic and the overhead of sharing group reconfiguration. Sharing Group Member Node (SGMN) was applied for sub-sharing group generation techniques with neighboring nodes at a 1-hop distance in order to reduce traffic for file sharing. The proposed technique achieved excellent results compared to a previous technique in transmission delay time, average data received time, and data transmission disconnection rate.

This fine collection of papers was achieved by fruitful collaborations. We wish to thank all the authors for their contributions and the reviewers for assisting our editorial work. We do hope that the papers included in this issue will satisfy the audience of the Wireless Personal Communications Journal and readers will find them interesting. Furthermore, we would like to thank Professor Ramjee Prasad, editor-in-chief of the international journals Wireless Personal Communications, for his valuable remarks and his undeterred help throughout the publication process. 


\section{References}

1. Jeong, Y. S., \& Shin, S. S. (2015). An efficient authentication scheme to protect user privacy in seamless big data services. Wireless Personal Communications. doi:10.1007/s11277-015-2990-1.

2. Lee, W., Cho, H., \& Shon, K. (2015). Systematical classification scheme management to provide efficient National R\&D Service in P2P. Wireless Personal Communications. doi:10.1007/s11277-0153111-X.

3. Lee, C., \& Lee, G. K. (2015). An alpha cut filter-based proxy caching control for wireless coverage streaming services. Wireless Personal Communications. doi:10.1007/s11277-015-3032-8.

4. Lee, S., \& Seo, K. K. (2015). A hybrid multi-criteria decision-making model for a cloud service selection problem using BSC, fuzzy Delphi method and fuzzy AHP. Wireless Personal Communications. doi:10.1007/s11277-015-2976-Z.

5. Lee, S., Lee, G., Lee, Y., \& Kim, J. (2015). A study on the improved normalized cut algorithm using a bilateral filter for efficient object extraction from image. Wireless Personal Communications. doi:10. 1007/s11277-015-3033-7.

6. Kim, D., Jung, S., Hwang, D. J., \& Kim, S. (2015). Mobile-based DoS attack security agent in sensor networking. Wireless Personal Communications. doi:10.1007/s11277-015-3098-3.

7. Jang, S. W., \& Jung, M. (2015). Detection of harmful content using multilevel verification in visual sensor data. Wireless Personal Communications. doi:10.1007/s11277-015-2966-1.

8. Lee, K., Seo, H., \& Lee, K. (2015). An adaptive asymmetric transmission scheme for MPEG4/SVC signals in MIMOOFDM system. Wireless Personal Communications. doi:10.1007/s11277-015-3064-0.

9. Choi, H., \& Sim, S. (2015). A study on efficiency of markup language using DOM tree. Wireless Personal Communications. doi:10.1007/s11277-015-3057-z.

10. Lee, H. S., Kwon, S. Y., \& Lim, J. H. (2015). A development of a lighting control system based on context-awareness for the improvement of learning efficiency in classroom. Wireless Personal Communications. doi:10.1007/s11277-015-2811-6.

11. Kim, J. C., Jung, H., Kim, S. H., \& Chung, K. Y. (2015). Slope based intelligent 3D disaster simulation using physics engine. Wireless Personal Communications. doi:10.1007/s11277-015-2788-1.

12. Lee, T. G., \& Lee, S. H. (2015). Dynamic bio-sensing process design in mobile wellness information system for smart healthcare. Wireless Personal Communications. doi:10.1007/s11277-015-2967-0.

13. Park, S. T., Im, H., \& Noh, K. S. (2015). A study on factors affecting the adoption of LTE mobile communication service: The case of South Korea. Wireless Personal Communications. doi:10.1007/ s11277-015-2802-7.

14. Park, S. T., Kim, Y. R., Jeong, S. P., Hong, C. I., \& Kang, T. G. (2015). A case study on effective technique of distributed data storage for big data processing in the wireless internet environment. Wireless Personal Communications. doi:10.1007/s11277-015-2794-3.

15. Kim, J., Chung, K. Y., \& Kang, M. A. (2015). Continuous gesture recognition using HLAC and lowdimensional space. Wireless Personal Communications. doi:10.1007/s11277-015-3068-9.

16. Yang, Y. W., Yu, W. H., \& Lim, H. S. (2015). Predicting second language proficiency level using linguistic cognitive task and machine learning techniques. Wireless Personal Communications. doi:10. 1007/s11277-015-3062-2.

17. Kim, S., \& Na, W. (2015). Safe data transmission architecture based on cloud for internet of things. Wireless Personal Communications. doi:10.1007/s11277-015-3063-1.

18. Jeong, K., \& Cho, H. J. (2015). A digitalized recomposition technique based on photo quality evaluation criteria. Wireless Personal Communications. doi:10.1007/s11277-015-2977-y.

19. Jung, H., \& Chung, K. Y. (2015). PHR based health index mobile service using decision support model. Wireless Personal Communications. doi:10.1007/s11277-015-3069-8.

20. Yang, H. S., \& Sun, J. H. (2015). A study on stable data transmission using hierarchical share group in mobile ad hoc network. Wireless Personal Communications. doi:10.1007/s11277-015-2882-4. 


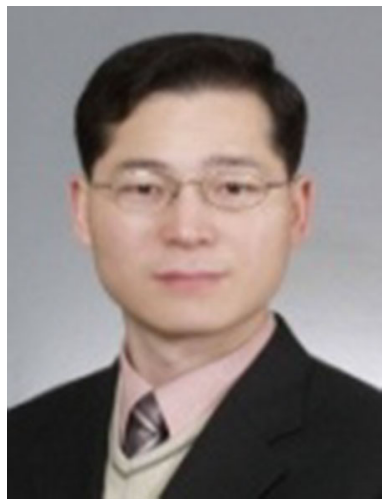

Sun-Moon Jo received the Ph.D. degrees from the Inha University, Korea, in 2007. He has worked for Seven System Korea. He is currently a professor in the Department of Computer Information Technology Education, Paichai University, Republic of Korea. His research interests include XML, Security, Knowledge System, HCI, Deep learning, and Recommendation. He serves as Director and a member of the Editorial Committee of the Korea Knowledge Information Technology Society, as Director of the Korea Contents Association, as Member of the Korean Society for Internet Information, as Member of the Korea Information Processing Society, as Member of the Institute of Electrical and Electronics Engineers, as Steering Committees of International Conference on Digital Policy and Management, and as Excellent Professor Award of the August 2015 Paichai University President. Also, he is editorial board members of several International Journals.

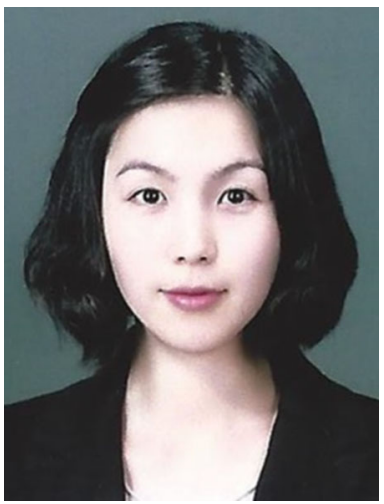

Yu-Keum Jeong is the executive secretary of Society of Digital Policy and Management, Republic of Korea. She supervised and managed conferences including International Conference on Digital Policy and Management, International Conference Convergence Technology, International Conference on Internet of Thing and Convergence, International Conference on Computer and Convergence Computing, and International Conference for Small and Medium Business. Her research topics are Contents Design, HCI, Intelligent System, IT Convergence, Ubiquitous Computing, Data Mining, Game Planning, and Recommendation. Furthermore, she has controlled special issues of prestigious international journals. Also, she is editorial board members of several International Journals indexed by SCOPUS.

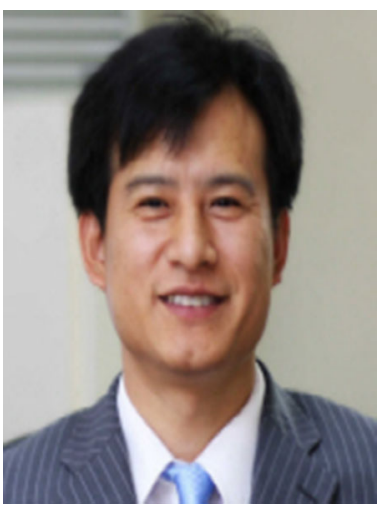

Jungsoo Han received a BS, an MS, and a Ph.D. in Computer Engineering from Kyung Hee University, Republic of Korea. Since 2001, he has been a Professor in the Division of Information and Communication, Baekseok University, Cheonan City, Chungnam, Republic of Korea. In 2014, he researched Convergence IT and Creative Education Methodology at California State University Fullerton as an Exchange Professor. His research topics include Data Mining, Contents Planning, 3D Modeling and CBD, Telemedicine, Knowledge-based Decision Support Systems, Intelligent Systems, Convergence, HCI, and Recommendation Systems. He has edited books on computer science and convergence technology. He serves as Executive Editing Director of the International Conference on Convergence Content (ICCC), as General Co-Chair of the International Conference on Digital Policy and Management (ICPDM), as General Co-Chair for steering committees of the International Conference on Convergence Technology (ICCT), as Workshop Chair of the International Conference on Information Science and Application 2013, as Workshop Chair of the 2th International Conference on IT Convergence and Security 2012, as Vice President of the Korea Contents Association, as Vice President and a member of the Editorial Committee of the Society of Digital Policy and Management, and as Vice President of the Editorial Committee of the Korea Contents Association. 